\title{
EXPOSURE TO A ROTATING VIRTUAL ENVIRONMENT DURING TREADMILL LOCOMOTION CAUSES ADAPTATION IN HEADING DIRECTION
}

\author{
Mulavara $\mathrm{AP}^{1}$, Richards $\mathrm{JT}^{2}$, Ruttley $\mathrm{T}^{3}$, Marshburn $\mathrm{A}^{3}$, Nomura $\mathrm{Y}^{4}$, Bloomberg $\mathrm{JJ}^{3}$ \\ ${ }^{1}$ National Space Biomedical Research Institute, Houston, TX, USA; ${ }^{2}$ Wyle Life Sciences, \\ Inc., Houston, TX 77058, USA; ${ }^{3}$ NASA Johnson Space Center, Houston, TX 77058, \\ USA; ${ }^{4}$ Nihon University School of Medicine, Tokyo, Japan 173-8610
}

\section{Corresponding Author:}

Ajitkumar P. Mulavara, Ph.D.

Senior Scientist

Neuroscience Laboratory

NASA- Johnson Space Center

NSBRI-Baylor College of Medicine

2101 NASA Parkway

Mail Code: SK/B272

Houston TX 77058

E-mail: ajitkumar.mulavara1@jsc.nasa.gov 


\section{ABSTRACT}

The goal of the present study was to investigate the adaptive effects of variation in the direction of optic flow, experienced during linear treadmill walking, on modifying locomotor trajectory. Subjects $(n=30)$ walked on a motorized linear treadmill at 4.0 $\mathrm{km} / \mathrm{h}$ for 24 minutes while viewing the interior of a $3 \mathrm{D}$ virtual scene projected onto a screen $1.5 \mathrm{~m}$ in front of them. The virtual scene depicted constant self-motion equivalent to either 1) walking around the perimeter of a room to one's left (Rotating Room group) 2) walking down the center of a hallway (Infinite Hallway group). The scene was static for the first 4 minutes, and then constant rate self-motion was simulated for the remaining 20 minutes. Before and after the treadmill locomotion adaptation period, subjects performed five stepping trials where in each trial they marched in place to the beat of a metronome at 90 steps/min while blindfolded in a quiet room. The subject's final heading direction (deg), final X (for-aft, $\mathrm{cm}$ ) and final Y (medio-lateral, $\mathrm{cm}$ ) positions were measured for each trial. During the treadmill locomotion adaptation period subject's $3 \mathrm{D}$ torso position was measured. We found that subjects in the Rotating Room group as compared to the Infinite Hallway group: 1) showed significantly greater deviation during post exposure testing in the heading direction and $\mathrm{Y}$ position opposite to the direction of optic flow experienced during treadmill walking 2) showed a significant monotonically increasing torso yaw angular rotation bias in the direction of optic flow during the treadmill adaptation exposure period. Subjects in both groups showed greater forward translation (in the $+\mathrm{X}$ direction) during the post treadmill stepping task that differed significantly from their pre exposure performance. Subjects in both groups reported no perceptual deviation in position during the stepping tasks. We infer that 
viewing simulated rotary self-motion during treadmill locomotion causes adaptive modification of sensory-motor integration in the control of position and trajectory during locomotion which functionally reflects adaptive changes in the integration of visual, vestibular, and proprioceptive cues. Such an adaptation in the control of position and heading direction during locomotion due to the congruence of sensory information demonstrates the potential for adaptive transfer between sensorimotor systems and suggests a common neural site for the processing and self-motion perception and concurrent adaptation in motor output. This will result in lack of subjects' perception of deviation of position and trajectory during the post treadmill step test while blind folded. 


\section{INTRODUCTION}

Vision, more precisely - optic flow, is a fundamental parameter that modulates motor output during locomotion (Gibson 1966). Optic flow, as an independent component of visual sensory input, is essential for controlling the estimation of distances, heading direction, dynamic balance and posture during locomotion (Warren et al. 1996; Bardy et al. 1999; Warren et al. 2001; Richards et al. 2004). Linear and rotating optic flow has been shown to cause directionally specific postural sway and positional deviations during treadmill and over ground walking (Warren et al. 1996; Keshner and Kenyon 2000). The rate at which people walk on a self-driven treadmill has been shown to depend on the velocity of an artificial optic flow pattern along the line of sight relative to their walking speed (Prokop et al. 1997). Linear optic flow has also been shown to cause directionally specific postural sway and positional deviations during treadmill locomotion (Bardy et al. 1996; Bardy et al. 1999; Jahn et al. 2001; Warren et al. 1996; Warren et al. 2001). The effects of rotational optic flow have also been demonstrated. When oscillated in roll and viewed during quiet stance, scenes containing complex, realistic content caused more postural sway than scenes with simple radial patterns (Duh et al. 2002). While walking over ground in a stereoscopic virtual environment that rotated in roll, subjects showed compensatory torso rotation in the direction of scene rotation that resulted in positional variation away from a desired linear path (Keshner and Kenyon 2000).

Humans can rapidly readjust and recalibrate various characteristics of their gross movement activities depending on the interactions between optic flow and biomechanical factors during locomotion (Rieser et al, 1990; Rieser et al. 1995). Some of these characteristics include the estimation of distances to walk to reach a target and the direction of movement. Investigations on the directional control of locomotion have 
shown that blindfolded subjects, after walking in place on a counterrotating platform or circular treadmill, while maintaining visual stationarity by keeping the torso and head orientations in the straight ahead direction, veered from their straight ahead trajectory in the same direction of the preceding turntable rotation (Gordon et al. 1995a; Gordon et al. 1995b; Weber et al. 1998; Earhart et al. 2001; Earhart et al. 2002; Weber et al. 2002). These authors have hypothesized that reinterpretation of intersegmental proprioceptive cues, triggered by the conflict between stationary vision and the turning feet on the platform, induces veering of locomotor trajectory from the straight ahead direction. Further experiments with concomitant optokinetic stimulation while stepping on a counter rotating platform caused a proportional increase in turning during post treadmill stepping in place (Jurgens et al. 1999). Jurgens, et al. (Jurgens et al. 1999) suggest that the previous result of adaptive modification of locomotor trajectory was the outcome of an adaptation of the somatosensory channel and not by a visual-somatosensory mismatch. However, subjects that are exposed to prolonged optokinetic stimulation, seated in the center of a rotating drum and watching a striped pattern, also generate curved walking trajectories in the direction opposite to that of the optokinetic stimulus when asked to step in place with their eyes closed (Kato et al. 1977; Gordon et al. 2003). Thus, optokinetic stimulation alone can cause an adaptive modification in the locomotion heading direction. In a series of experiments, Rieser, et al. (Rieser et al. 1990; Rieser et al. 1995) demonstrated that humans can reliably estimate the distance to a target and walk to it without vision. Importantly, this capability can be re calibrated after being exposed to a new relationship between the rates of walking relative to that of the optical flow of the surrounding environment (Rieser et al. 1995). Thus, humans have a flexible perceptual 
motor system that has the ability to learn the covariation between the optical flow and the consequence of their walking in the environment (Rieser et al. 1995).

The goal of this study was to determine if plastic adaptive modification in locomotor trajectory, during a stepping task, could be achieved when exposed to a new relationship between the direction of optic flow, with matched optic flow rate, and that of walking during linear treadmill locomotion. Hence, subjects were tested using a stepping in place test pre and post exposure to optic flow that either rotated about the subjects' vertical axis (yaw optic flow rotation) or translated fore and aft in the direction of linear locomotion. Further, this study also aimed to investigate the underlying mechanisms of adaptation by measuring torso kinematics during the adaptive exposure period.

\section{METHODS}

\section{Participants}

30 healthy subjects of average $(( \pm 1 \mathrm{SD})$ age $=32.0( \pm 1.3)$ yr., height $=167.4( \pm 4.4)$ $\mathrm{cm}$, and weight $=67.2 \pm 4.6 \mathrm{~kg}$ with normal or corrected-to-normal vision were recruited from the Human Test Subject Facility at Johnson Space Center (JSC) in Houston, TX. The experimental protocol was approved by the NASA-JSC Committee for the Protection of Human Subjects (CPHS), and informed consent was obtained prior to testing.

\section{Visual Scene Adaptation Protocol}

Prior to experimental trials, subjects walked on the treadmill until they were comfortable walking without holding onto the handrails (usually $<5$ minutes). Subjects walked at 4.0 $\mathrm{km} / \mathrm{h}$ while viewing either the Rotating Room (RR group, Fig $1 \mathrm{~A}$ ) or the Infinite Corridor 
(IC group, Fig 1B) visual scene for 20 minutes continuously. An equal number of subjects were randomly assigned to the two groups. The scenes either rotated in yaw in the clockwise direction at a constant rate of $30 \%$ for the RR group or translated from fore to aft at a rate of $4 \mathrm{~km} / \mathrm{h}$ for the IC group. The rotation rate of the Rotating Room visual scene was chosen such that the forward translation component of optic flow was equivalent to that seen in the Infinite Corridor visual scene. Previous studies have shown rotation rates close to this to be effective in causing sensations of self-motion (Watt et al. 1993; Allison et al. 1999). Data were collected during 120-sec epochs of time uniformly distributed during the walk period. The first data take while subjects walked on the treadmill included data obtained $1 \mathrm{~min}$. before the scene started rotating while the scene was static (static epoch) and 1 min. after the scene started rotating (rotating epoch). The remainder of the data collection was uniformly timed through the remaining walk period. Subjects were instructed to fixate on the scene for the entire trial and try to immerse themselves in it without paying attention to their position on the treadmill belt. A spotter provided guiding commands (e.g., "move left") when the subject approached the limits of the treadmill belt surface.

\section{Visual Optic Flow Scenes}

The monoscopic, passive-immersive 3D visual scenes were created using graphic modeling software (3ds max 4; Discreet, Montreal, Que.) and rendered using virtual environment software (VRUT v. 2.5, Python v. 2.0) on a PC computer (2.2 GHz, Intel Pentium 4 processor, nVIDIA Quadro2 EX graphics card). Scenes either constantly rotated in yaw or constantly translated along the anterior-posterior (AP) body axis. The 
rotating scene, the Rotating Room (Fig. 1A), was a cubical room with simulated dimensions $12 \times 12 \times 6 \mathrm{~m}$ [length (line of sight) $\times$ width $\times$ height]. The Rotating Room was rich in polarizing visual content, including distinctive markings on the floor and ceiling and realistic texture-mapped objects such as trees, desks, chairs, and gravity-cued pictures. The subject's simulated eyepoint was displaced $7.0 \mathrm{~m}$ to the right and $3.0 \mathrm{~m}$ back from the center of the Rotating Room as it rotated aboul an axis coincident with its center in the clockwise direction, creating the illusion that the subject was walking around the perimeter of the room to his or her left. The second scene, the Infinite Corridor (Fig. 1B), was a rectangular chamber $6 \mathrm{~m}$ wide, $6 \mathrm{~m}$ high, and "infinitely" long with polarized content similar to that in the Rotating Room. The subject's simulated eyepoint was placed $1.82 \mathrm{~m}$ above the floor and centered between the left and right walls while the hallway interior constantly translated such that it appeared as if the subject was walking down a hallway at a fixed rate. Simulated scene dimensions were chosen in order to maximize desired perceptual effects as determined from pilot studies.

\section{Stepping Task Protocol}

To determine the effects of exposure to the adaptation protocol on locomotor trajectory, subjects completed five stepping trials both before and after the visual scene adaptation period. While blindfolded, subjects were instructed to march in place to the sound of a metronome beating at 90 steps per minute for a total of 100 steps per trial. At the end of each trial, the subject's final position (X - fore/aft, Y- left/right) relative to the initial position $(0 \mathrm{~cm}, 0 \mathrm{~cm})$ and the intercepts of the line $\left(X_{\mathrm{int}}, Y_{\mathrm{int}}\right)$ indicating the 
heading orientation direction with respect to the initial heading orientation direction (straight ahead $=00 \mathrm{deg}$ ) were measured (see Fig. 2). Subjects were then walked back while still blind folded by a spotter to the origin in a random, winding pattern such that they were facing the same direction (feet parallel and toes pointing forward) at the beginning of each trial. Thus, subjects never received post-test feedback of their performance and final heading direction. Subjects performed the stepping task immediately after completing the 20 min visual scene adaptation period.

\section{Experimental Setup}

Scenes were back-projected onto a screen positioned $1.5 \mathrm{~m}$ in front of the subject's eye point using a digital DP1100 projector (Kodak, Inc., Rochester, NY). Projected scenes subtended a $65^{\circ} \mathrm{H} \mathrm{x} 48^{\circ} \mathrm{V}$ field-of-view (FOV) and were viewed by subjects while walking on a motorized treadmill (Trotter Instrument Corp., Amherst, NY) with a surface area of $51 \times 156 \mathrm{~cm}$. To prevent injury through falling, each subject wore a full-body harness that was attached to an overhead gantry that shut the treadmill off if the subject fell. During nominal performance this harness provided no support and did not interfere with the natural movement of the trunk or limbs. The subjects wore headmounted sport goggles (with plastic lens removed) that occluded view of peripheral objects (e.g., support-harness gantry) to eliminate orientation cues and help maximize immersion in the virtual scene. Subjects were also instructed to do their best to immerse themselves in the virtual scene. Foot-switches with force sensing resistors (Motion LabSystems, Inc., Baton Rouge, LA) were attached to the plantar surface of each shoe at the heel and toe and were sampled at $1000 \mathrm{~Hz}$. These were used to define the temporal 
aspects of the gait cycle. A thumb on-off switch was used by the operator to indicate the onset of scene rotation and was sampled by the footswitch data acquisition system.

Before each testing session, passive lightweight retro-reflective markers that served as tracking landmarks were placed on the torso to measure its 6 degree-of-freedom (DOF) movements while subjects walked on the treadmill viewing the visual scenes in front of them. A T-shaped vest with markers placed on the superior tip of the spinous process of the $\mathrm{C} 7$ vertebrae and at equal distances laterally from the midline at the level of the 10th thoracic vertebrae was worn about the subject's torso. Previous studies (Keshner et al. 1988) have shown that trunk motion can be used to characterize dynamic postural stability during locomotion.

Three-dimensional motion data were recorded using a video-based motion measurement system (Motion Analysis Corp., Santa Rosa, CA) with respect to the origin coincident with the surface of the treadmill. Three time-synchronized CCD cameras were used for data collection with a sampling rate of $60 \mathrm{~Hz}$. The resolution and repeatability were computed to $0.1 \mathrm{~mm}$, and accuracy ranged from $0.05 \mathrm{~mm}$ to $0.16 \mathrm{~mm}$ (Miller et al. 2002; Mulavara et al. 2002; Richards et al. 2004).

\section{DATA ANALYSIS}

\section{Pre-post scene exposure}

The recorded final positions $(X, Y)$ and the intercepts of the line $\left(X_{\text {int }}, Y_{\text {int }}\right)$ indicating the heading orientation direction, were used to calculate the heading orientation direction angle (HD). The HD was calculated and coded for direction of movement as follows: 


$$
\begin{aligned}
& \Theta_{C W}=\Theta^{*}-\frac{\Pi}{2}, X>0 \\
& \Theta_{C W}=\Theta^{*}-\frac{3 \Pi}{2}, X<0 \\
& \Theta_{C C W}=\Theta^{*}+\frac{3 \Pi}{2}, X>0 \\
& \Theta_{C C W}=\Theta^{*}+\frac{\Pi}{2}, X<0 \\
& \Theta^{*}=a \tan \left(\frac{Y-Y_{\text {int }}}{X-X_{\text {int }}}\right)
\end{aligned}
$$

where, $\mathrm{CW}$ and $\mathrm{CCW}$ are the clockwise and anticlockwise directions of subject

movement with respect to their initial positions. These data obtained during the pre and post adaptation step tests for the two groups of subjects were collated and subjected to a repeated measures ANOVA (Within subject factors - Adaptation, 2 levels: pre and post; vs Across subject factor - Group: two levels - RR and IC). A student t- test was performed on the difference between the pre and post adaptation measures for the three variables. Data were analyzed at a significance level of .05 using a standard statistical software package (SPSS v. 10.0, Chicago, IL).

\section{Torso kinematics obtained during the visual scene adaptation protocol}

Marker data were processed to derive three-dimensional position information relative to a coordinate frame coincident with the surface of the treadmill. Thus, subjects walked toward the $+\mathrm{X}$ direction and the belt moved in the $-\mathrm{X}$ direction, the vertical axis orthogonal to the surface of the treadmill was $+Z$, and the $Y$ axis was orthogonal to the $\mathrm{X}-\mathrm{Z}$ plane. The marker trajectories were filtered using a fourth-order, low-pass, zerophase-response, Butterworth filter with a cut-off frequency at $5 \mathrm{~Hz}$. Torso body axis 
rotation in the yaw plane (i.e. rotation about the $Z$ global axes) was calculated using an Euler angle convention (Chao 1980; Mulavara et al. 2002). The torso yaw angular orientation time series data were then broken up into consecutive gait cycles (right hecl strike to following right heel strike) using the footswitch data within each epoch of each trial. The torso yaw angular orientation time series during the rotating epoch was normalized by subtracting the average torso orientation during the static epoch. The magnitude and time at which maxima and minima of torso yaw rotation data during each gait cycle were calculated. A linear regression analysis was performed, for each subject in each group, using time as the independent variable and the torso maxima and minima as dependent variables, respectively, to evaluate the torso orientation bias during the adaptation period. Further, a single factor multivariate analysis of variance was performed on the torso maxima and minima slope ( $\mathrm{deg} / \mathrm{min}$ ) values across each group to test the hypothesis that during the adaptation period there was no significant difference between the torso orientation bias for the two groups of subjects. Data were analyzed at a significance level of 0.05 using a standard statistical software package (SPSS v. 10.0, Chicago, IL).

\section{RESULTS}

\section{Pre post scene exposure}

Figure 3 shows the average ( \pm one standard error of the mean) of the final heading direction (HD, deg; Figure 3A), final mediolateral (Y, cm; Figure 3B) position and the final fore-aft position (X, cm; Figure 3C) across all subjects pre and post exposure to the visual scene adaptation protocol for the two groups (RR and IC). Prior to the optic flow 
exposure, individually subjects varied their heading direction showing a tendency to deviate in both directions with the average over the five trials and across all subjects showing an overall tendency to deviate to the right which was not significantly different between the two groups. However, after being exposed to 20 minutes of optic flow during linear treadmill walking subjects in the IC group showed a tendency to deviate further to their right that was significantly different from the subjects in the RR group who deviated to their left. A repeated measures ANOVA on HD and Y position revealed there was a significant interaction for Group * Adaptation [HD: $\mathrm{F}_{(1,27)}=5.192, \mathrm{p}<0.031$; $Y$ position: $\left.F_{(1,29)}=5.208, p<0.030\right]$. However, there was no significant main effect of Adaptation for $\mathrm{HD}$ and $\mathrm{Y}$ position $(\mathrm{p}>0.05)$. A student $\mathrm{t}-$ test on the difference between the pre and post adaptation $\mathrm{HD}$ and $\mathrm{Y}$ position revealed a significant difference between the two groups $(\mathrm{p}<0.05)$. Therefore, subjects in the RR group, exposed to the rotating optic flow in yaw, showed greater deviation in locomotor trajectory opposite to the direction of optic flow during post exposure stepping that significantly differed from those in the IC group. Moreover, subjects in both groups reported no perception of deviation from their start positions.

Repeated measures ANOVA on $\mathrm{X}$ position revealed there was a significant main effect of Adaptation $\left[\mathrm{F}_{(1,29)}=19.225, \mathrm{p}<0.0001\right]$. However, there was no significant interaction for Group * Adaptation for X position ( $p>0.05)$. Therefore, subjects in both the RR and IC groups, exposed to the rotating optic flow in yaw or translating fore-aft optic flow, showed greater forward translation during post exposure stepping.

\section{Torso kinematics obtained during the visual scene adaptation protocol}


Figures 4(A-D) shows the linear curve fit over the maxima and minima torso yaw angular position of all subjects while walking on the treadmill during the 20 minute exposure to the two moving visual scenes. Figure 5 shows the average ( \pm one standard error of the mean) slope of the linear regression of the maxima and minima torso yaw angular position of all subjects for the RR and IC groups. The multivariate single factor ANOVA on torso minima and maxima revealed a significant difference between the groups [torso minima: $F_{(1,22)}=31.209, p<0.0005$; torso maxima: $F_{(1,22)}=13.576, p$ $<0.001]$. Therefore, subjects in the RR group, exposed to the rotating optic flow in yaw, showed greater torso yaw rotation bias to the right with respect to space during the exposure while walking on the treadmill than those in the IC group.

\section{DISCUSSION}

In this study, subjects walking on a linear treadmill were exposed to an optic flow pattern that matched their linear translation speed but varied in the direction of rotation. Subjects were instructed to fully immerse themselves in the virtual environment inducing self motion sensations of walking straight along the long corridor (IC Group) or walking around the room to their left (RR Group). Subjects were asked to perform a controlled stepping test to measure adaptive changes in locomotor trajectory pre and post exposure to the optic flow stimuli.

\section{Pre and post scene exposure}

The HD and Y position results indicate that during the post treadmill stepping task subjects in the RR group, viewing the rotating optic flow, tended to deviate in the 
direction of the simulated self motion and opposite to the direction of optic flow, without any percept of rotation or deviation from their starting position. This was significantly different from the response seen with the subjects in the IC group during the post treadmill stepping task, wherein these subjects tended to step in place resulting in deviation in their heading direction that was not significantly different from their behavior prior to adaptive exposure. These results are similar to that of Gordon et al. (Gordon et al. 2003) and Kato, et al. (Kato et al. 1977). In these experiments, subjects performed a controlled stepping test before and after viewing moving black and white strip patterns while seated (optokinetic stimulation alone) rotating at the rate of 45 $\mathrm{deg} / \mathrm{sec}$ or $30 \mathrm{deg} / \mathrm{sec}$ for periods of $150 \mathrm{sec}$ and $30 \mathrm{~min}$ durations, respectively. Both studies showed that subjects tended to deviate in a direction opposite to the direction of optic flow stimulus during post exposure stepping task. Our experiments confirm that viewing a rotating optic flow in a virtual environment for 20 min. during linear treadmill walking also causes an adaptive response that modifies the trajectory of locomotion during stepping in place while blindfolded in a direction opposite to the optical stimulus direction.

The significant effect of the Adaptation factor in the $\mathrm{X}$ direction measure during post treadmill stepping task in subjects from both groups shows that treadmill locomotion while viewing realistic optic flow with matched speed regardless of direction results in a recalibration of the visuomotor system controlling the ability to remain in one place. Such a recalibration have been shown previously when subjects attempted to walk in place after running on a treadmill or behind a moving golf cart without vision (Anstis 1995; Durgin and Pelah 1999). 


\section{Torso kinematics obtained during the visual scene adaptation protocol}

The results of our study indicate that subjects in the RR group, introduce a monotonically increasing bias in their torso yaw rotation with respect to space in the direction of the optokinetic stimulation over the period of exposure which is significantly different from those in the IC group where no significant bias in torso rotation was observed. Such a torso rotation may indeed help maintain stability to continue walking on the linear treadmill when subjects are immersed in the self motion of walking around the comer in a virtual room towards their left. However, the bias of torso position re space may also introduce a bias with respect to the foot motion and cause an adaptation of the intersegmental proprioceptive signal in terms of the heading direction similar to those reported in the podokinetic adaptation experiments (Gordon et al. 1995a; Gordon et al. 1995b; Weber et al. 1998; Jurgens et al. 1999). Further, in the podokinetic adaptation experiments of Jurgens, et al. (Jurgens et al. 1999) subjects showed an additive response to the podokinetic and visual optokinetic rotation. It should be noted that in these podokinetic adaptation experiments, subjects were asked to walk on a circular rotating treadmill or rotating drum by keeping their head and torso in the straight ahead direction while they counter rotated their foot to the support surface motion to maintain their position on the moving surface. In the present study, the bias in the torso yaw angular position relative to the spatial straight ahead direction was introduced when subjects were walking on a linear treadmill and viewing a rotating optokinetic flow pattern about the yaw axis. It should also be noted that in the present study, subjects fixated their gaze on the imposed virtual environment directly in front of them allowing them to fully 
experience self motion during the visual scene exposure period. Such a disposition of holding the head to fixate gaze in the straight ahead direction with respect to space will also result in a head re torso bias that is monotonically increasing through the exposure period. Thus, subjects walked along the direction of the linear treadmill belt (fore-aft motion) with the feet moving in the same direction along with it and their heads fixated on the visual optic flow presented in front of them while their torso was rotated about their vertical axis and biased in the direction of the optic flow. This may result in the rotated perception of their straight ahead via the linear summation of the vestibular and proprioceptive signals (Mergner et al. 1991; Mergner et al. 1993).

\section{Common neural site estimating and controlling heading direction during locomotion}

The present results show that viewing simulated rotary self-motion during linear treadmill locomotion causes adaptive modification of sensory-motor integration for estimating the trajectory during post treadmill stepping tasks. Such an adaptation of the heading direction is accompanied by a lack of subjects perception of deviation of position during the post treadmill step test while blind folded. Similar lack of perception of changes in locomotor trajectory have been reported in the literature after the podokinetic adaptation paradigm as well as after the exposure to rotating optic flow while seated during the post adaptation stepping tasks (Gordon et al. 1995a; Gordon et al. 1995b; Weber et al. 1998; Gordon et al. 2003; Jurgens et al. 1999). Previous works on the mechanisms involved in the estimation of self turning in the dark have resulted in the hypothesis of fusion of sensory information from multiple modalities (Jurgens et al. 1999). A number of studies have shown the transfer of adaptation from the systems 
interpreting optic flow to the motor system. There are numerous examples of optokinetic stimulus causing modification of gain of the vestibulo-ocular reflex (Schor and Westall 1986) and modification of the VOR affecting the gain of the optokinetic nystagmus response (Aoki and Yagi 1988). Also, previous research on a visuomotor task of pointing has shown a transfer of adaptation after modifying the gain of the VOR (Bloomberg et al. 2000). These studies demonstrate the potential for adaptive transfer between various motor systems and suggest a common neural site for the processing and self-motion perception and concurrent adaptation in motor output.

Various cortical areas have been implicated in the processing of this synergetic interplay between optic flow and other sensory modalities. The dorsal stream of primate cortex contains motion processing areas that are selective for optic flow and self-motion (Lappe et al. 1999). One such functionally specialized area is the ventral intraparietal area (VIP) within the posterior parietal cortex of the primate (Bremmer et al. 2001). The VIP contains many neurons that show directionally selective discharges to stimuli from multiple modalities including moving visual, tactile, vestibular, or auditory stimuli. Functional imaging data on humans also reveal a network of cortical areas, one of which is located in the depth of human intraparietal sulcus, that respond to multimodal stimuli conveying motion information (Bremmer et al. 2001). Accordingly, these authors have suggested that this area constitutes the human equivalent of primate area VIP. Studies of monkeys strongly suggested that VIP responds to optic flow and is involved in the encoding of self motion and heading direction (Bremmer et al. 2002; Schlack et al. 2002; Zhang and Britten 2004; Zhang et al. 2004). In addition to VIP, the medial superior temporal area of extrastriate cortex (MST) is suggested to contain sufficient information 
for threshold judgments of optic flow (Heuer and Britten 2004), and is suggested to have a role in processing of heading direction and perception of self motion (Britten and van Wezel 1998; Britten and Van Wezel 2002; Bremmer et al. 1999). These cortical areas maybe involved in providing information for locomotor heading direction and may be involved in the adaptive transfer between motor output modalities. Therefore, vision, vestibular and somatosensory information may be processed as a weighted sum and therefore produce a perceived change in heading direction when the central interpretation of one of the sensory channels is modified. Importantly, this change in heading direction may not be perceived by other sensory modalities (i.e. vestibular) because of central reinterpretation of self-motion perception induced by exposure to optic flow.

\section{ACKNOWLEDGEMENTS}

This study was supported in part by NASA and through NASA Cooperative Agreement NCC9-58 with the National Space Biomedical Research Institute by grants to Jacob J Bloomberg (PI). We also wish to thank the subjects for contributing their time participating in this study.

\section{REFERENCES}

Allison RS, Howard IP, Zacher JE (1999) Effect of field size, head motion, and rotational velocity on roll vection and illusory self-tilt in a tumbling room. Perception 28 : 299-306

Anstis S (1995) Aftereffects from jogging. Exp Brain Res 103: 476-478

Aoki H, Yagi T (1988) Effect of VOR gain changes on OKR gain control in human subjects. Adv Otorhinolaryngol 41: 58-62 
Bardy BG, Warren WH, Jr., Kay BA (1996) Motion parallax is used to control postural sway during walking. Exp Brain Res 111: 271-282

Bardy BG, Warren WH, Jr., Kay BA (1999) The role of central and peripheral vision in postural control during walking. Percept Psychophys 61: 1356-1368

Bloomberg JJ, Merkle LA, Barry SR, Huebner WP, Cohen HS, Mueller SA, Fordice J (2000) Effects of adaptation of vestibulo-ocular reflex function on manual target localization. J Vestib Res 10: 75-86

Bremmer F, Duhamel JR, Ben Hamed S, Graf W (2002) Heading encoding in the macaque ventral intraparietal area (VIP). Eur J Neurosci 16: 1554-1568

Bremmer F, Kubischik M, Pekel M, Lappe M, Hoffmann KP (1999) Linear vestibular self-motion signals in monkey medial superior temporal area. Ann N Y Acad Sci 871: $272-281$

Bremmer F, Schlack A, Duhamel JR, Graf W, Fink GR (2001) Space coding in primate posterior parietal cortex. Neuroimage 14: S46-51

Britten KH, van Wezel RJ (1998) Electrical microstimulation of cortical area MST biases heading perception in monkeys. Nat Neurosci 1: 59-63

Britten KH, Van Wezel RJ (2002) Area MST and heading perception in macaque monkeys. Cereb Cortex 12: 692-701

Chao EY (1980) Justification of triaxial goniometer for the measurement of joint rotation. J Biomech 13: 989-1006

Duh HB, Lin JJ, Kenyon RV, Parker DE, Furness TA (2002) Effects of characteristics of image quality in an immersive environment. Presence Teleoper Virtual Environ $11: 324-332$ 
Durgin FH, Pelah A (1999) Visuomotor adaptation without vision? Exp Brain Res 127:

$12-18$

Earhart GM, Jones GM, Horak FB, Block EW, Weber KD, Fletcher WA (2001) Forward versus backward walking: transfer of podokinetic adaptation. J Neurophysiol 86: $1666-1670$

Earhart GM, Jones GM, Horak FB, Block EW, Weber KD, Flctcher WA (2002) Podokinetic after-rotation following unilateral and bilateral podokinetic stimulation. J Neurophysiol 87: 1138-1141

Gibson J (1966) The senses considered as perceptual systems. Houghton Mifflin, Boston Gordon CR, Fletcher WA, Jones GM, Block EW (1995a) Is the stepping test a specific indicator of vestibulospinal function? Neurology 45: 2035-2037

Gordon CR, Fletcher WA, Melvill Jones G, Block EW (1995b) Adaptive plasticity in the control of locomotor trajectory. Exp Brain Res 102: 540-545

Gordon CR, Tal D, Gadoth N, Shupak A (2003) Prolonged optokinetic stimulation generates podokinetic after rotation. Ann N Y Acad Sci 1004: 297-302

Heuer HW, Britten KH (2004) Optic flow signals in extrastriate area MST: comparison of perceptual and neuronal sensitivity. J Neurophysiol 91: 1314-1326

Jahn K, Strupp M, Schneider E, Dieterich M, Brandt T (2001). Visually induced gait deviations during different locomotion speeds. Exp Brain Res 141: 370-374

Jurgens R, Boss T, Becker W (1999) Podokinetic after-rotation does not depend on sensory conflict. Exp Brain Res 128: 563-567 
Kato I, Miyoshi T, Pfaltz CR (1977) Studies on habituation of vestibulospinal reflexes. Effects of repetitive optokinetic and vestibular stimuli upon the stepping test. ORL J Otorhinolaryngol Relat Spec 39: 195-202

Keshner EA, Kenyon RV (2000) The influence of an immersive virtual environment on the segmental organization of postural stabilizing responses. J Vestib Res 10:207219

Keshner EA, Woollacott MH, Debu B (1988) Neck, trunk and limb muscle responses during postural perturbations in humans. Exp Brain Res 71: 455-466

Lappe M, Bremmer F, van den Berg AV (1999) Perception of self-motion from visual flow. Trends Cogn Sci 3: 329-336

Mergner T, Hlavacka F, Schweigart G (1993) Interaction of vestibular and proprioceptive inputs. J Vestib Res 3: 41-57

Mergner T, Siebold C, Schweigart G, Becker W (1991) Human perception of horizontal trunk and head rotation in space during vestibular and neck stimulation. Exp Brain Res 85: 389-404

Miller C, Mulavara A, Bloomberg J (2002) A quasi-static method for determining the characteristics of a motion capture camera system in a "split-volume" configuration. Gait Posture 16: 283-287

Mulavara AP, Bloomberg JJ (2002) Identifying head-trunk and lower limb contributions to gaze stabilization during locomotion. J Vestib Res 12: 255-269

Mulavara AP, Verstraete MC, Bloomberg JJ (2002) Modulation of head movement control in humans during treadmill walking. Gait Posture 16: 271-282 
Prokop T, Schubert M, Berger W (1997) Visual influence on human locomotion. Modulation to changes in optic flow. Exp Brain Res 114: 63-70

Richards JT, Mulavara AP, Bloomberg JJ (2004) Postural stability during treadmill locomotion as a function of the visual polarity and rotation of a three-dimensional environment. Presence:Teleoperations and Virtual Reality 13: 371-384

Rieser JJ, Ashmead DH, Talor CR, Youngquist GA (1990) Visual perception and the guidance of locomotion without vision to previously seen targets. Perception 19: $675-689$

Rieser JJ, Pick HL, Jr., Ashmead DH, Garing AE (1995) Calibration of human locomotion and models of perceptual-motor organization. J Exp Psychol Hum Percept Perform 21: 480-497

Schlack A, Hoffmann KP, Bremmer F (2002) Interaction of linear vestibular and visual stimulation in the macaque ventral intraparietal area (VIP). Eur J Neurosci 16: $1877-1886$

Schor C, Westall C (1986) Rapid adaptation of the vestibulo-ocular reflex and induced self-motion perception. Percept Psychophys 40: 1-8

Warren WH, Jr., Kay BA, Zosh WD, Duchon AP, Sahuc S (2001) Optic flow is used to control human walking. Nat Neurosci 4: 213-216

Warren WH, Kay BA, Yilmaz EH (1996) Visual control of posture during walking: functional specificity. J Exp Psychol Hum Percept Perform 22: 818-838

Watt D, Landolt J, Young L (1993) Effects of long term weightlessness on roll circularvection. Canadian Aeronautics Space Journal 39: 52-55 
Weber KD, Fletcher WA, Gordon CR, Melvill Jones G, Block EW (1998) Motor learning in the "podokinetic" system and its role in spatial orientation during locomotion. Exp Brain Res 120: 377-385

Weber KD, Fletcher WA, Melvill Jones G, Block EW (2002) Podokinetic after-rotation in patients with compensated unilateral vestibular ablation. Exp Brain Res 147: $554-557$

Zhang T, Britten KH (2004) Clustering of selectivity for optic flow in the ventral intraparietal area. Neuroreport 15: 1941-1945

Zhang T, Heuer HW, Britten KH (2004) Parietal area VIP neuronal responses to heading stimuli are encoded in head-centered coordinates. Neuron 42: 993-1001 


\section{Figure Legends}

Figure 1: Subjects viewed either the Rotating Room (RR group, A) rotating in yaw in the clockwise direction or the Infinite Comidor (IC group, B) translating from fore to aft at constant velocity during the rotating epoch of the visual scene adaptation protocol.

Figure 2: Axes orientation and measures calculated during the stepping task protocol: the subject's final position $(\mathrm{X}$ - fore/aft, $\mathrm{Y}$ - left/right) relative to the initial position $(0 \mathrm{~cm}, 0$ $\mathrm{cm}$ ) and the heading direction with respect to the initial heading orientation direction (straight ahead $=00 \mathrm{deg}$ ).

Figure 3: The average ( \pm one standard error of the mean) of the final heading direction $(\mathrm{HD}, \operatorname{deg} ; \mathrm{A})$, final mediolateral $(\mathrm{Y}, \mathrm{cm} ; \mathrm{B})$ position and the final fore-aft position $(\mathrm{X}$, $\mathrm{cm}$; C) across all subjects pre and post exposure to the visual scene adaptation protocol for the RR group (closed squares, ) and the IC group (closed diamond, _).

Figure 4: The linear curve fit over the minima ( $\mathrm{A}$ and $\mathrm{C})$ and maxima $(\mathrm{B}$ and $\mathrm{D})$ torso yaw angular position of all subjects while walking on the treadmill during the 20 minute exposure to the visual scene adaptation protocol for the IC group (A and B) and the RR group ( $\mathrm{C}$ and $\mathrm{D})$. The torso maxima and minima data during the rotating epoch, which was normalized with respect to the static epoch, is shown. 
Figure 5: The average ( \pm one standard error of the mean) slope of the linear regression of the maxima (closed squares, _) and minima (closed diamond, ) torso yaw angular position of all subjects for the RR (Rotating) and IC (Hallway) groups. 
A.

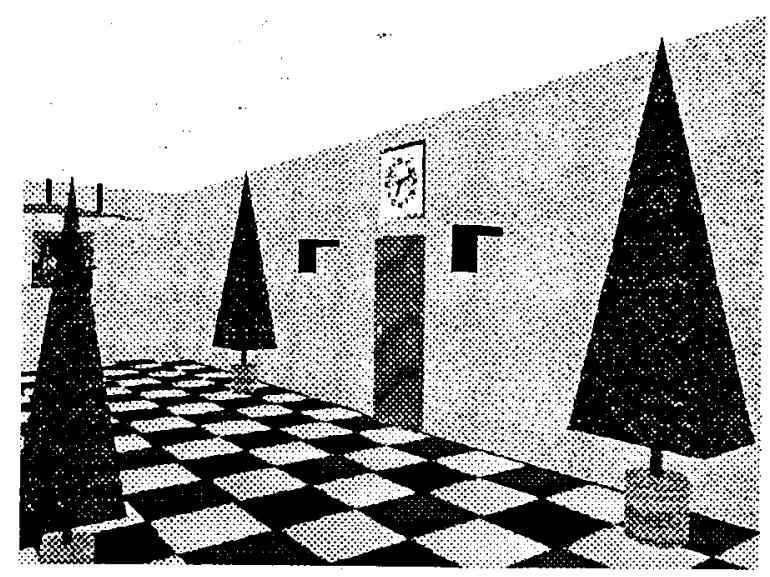

B.

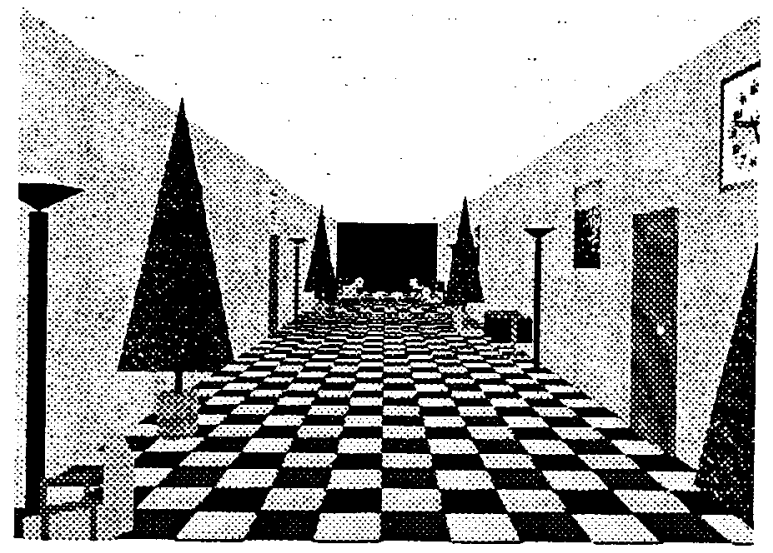

Figure 1 


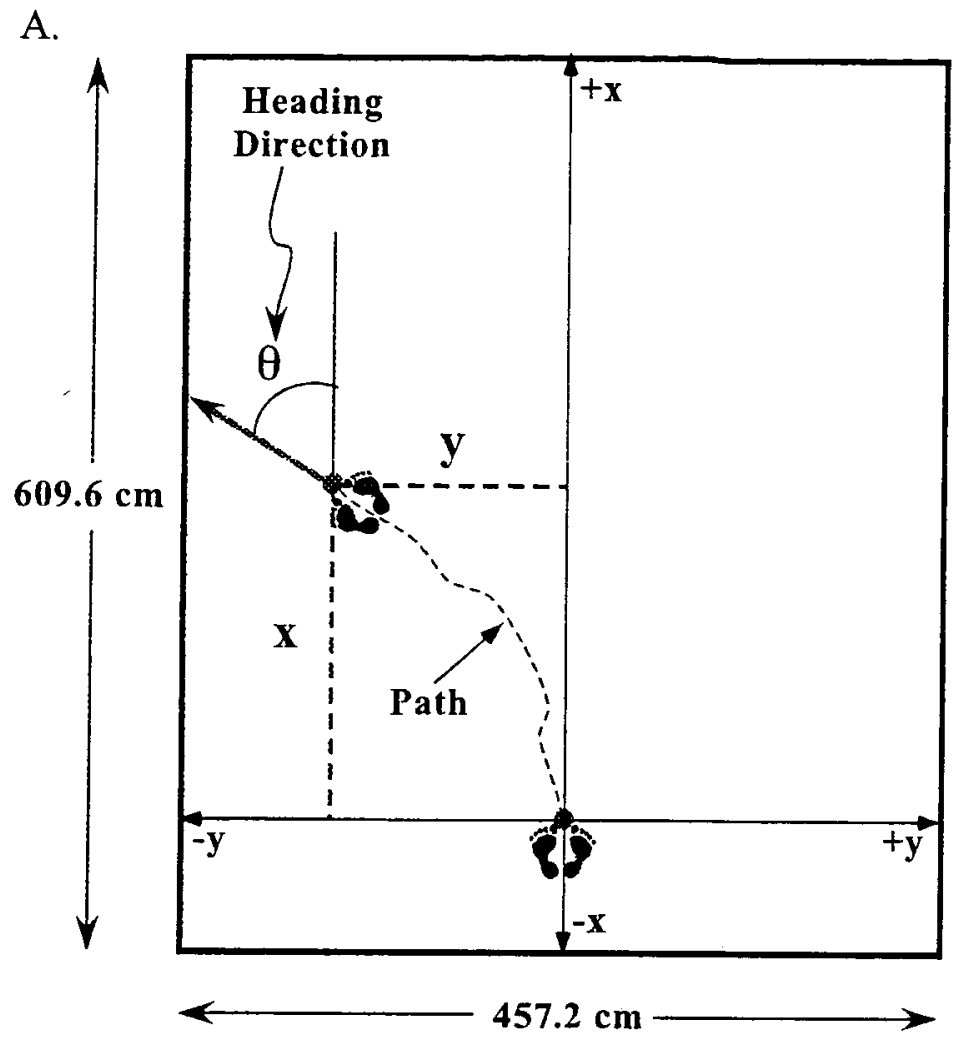

Figure 2 
A.

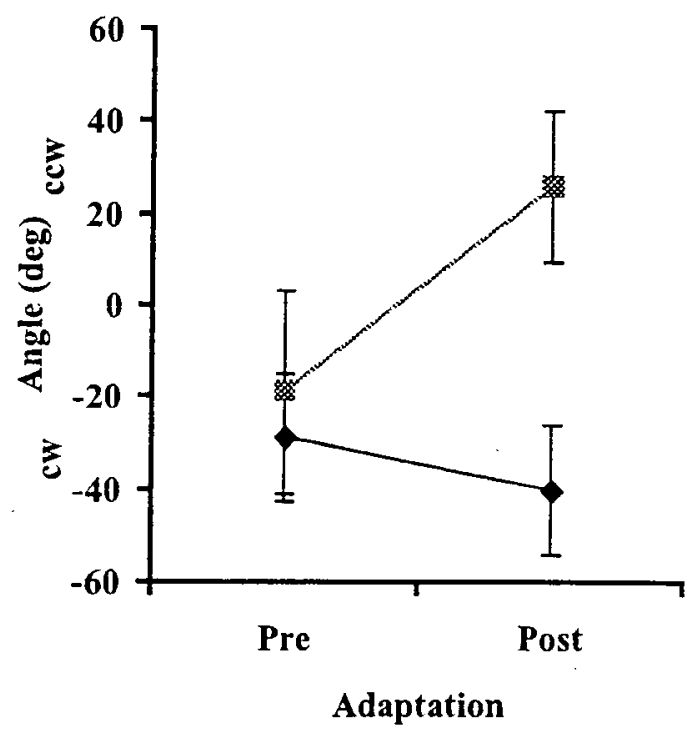

B.

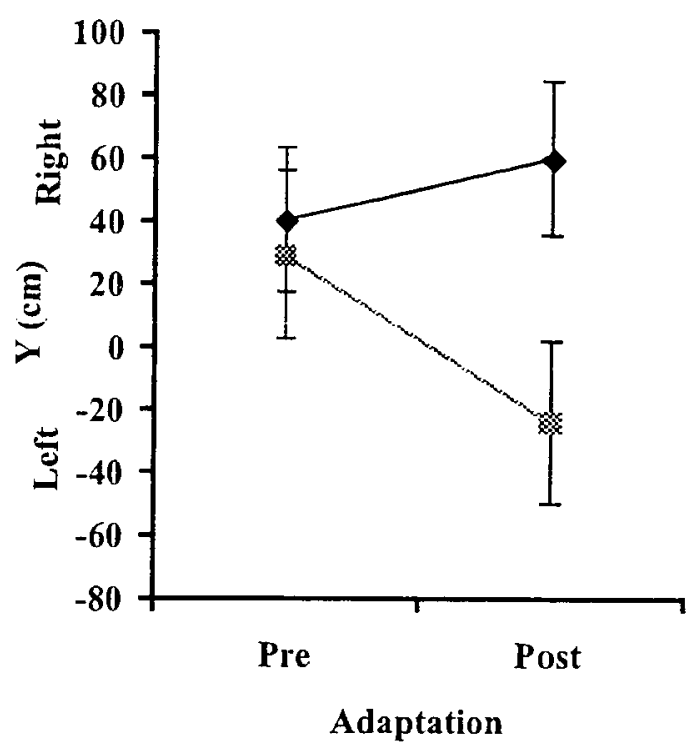

C.

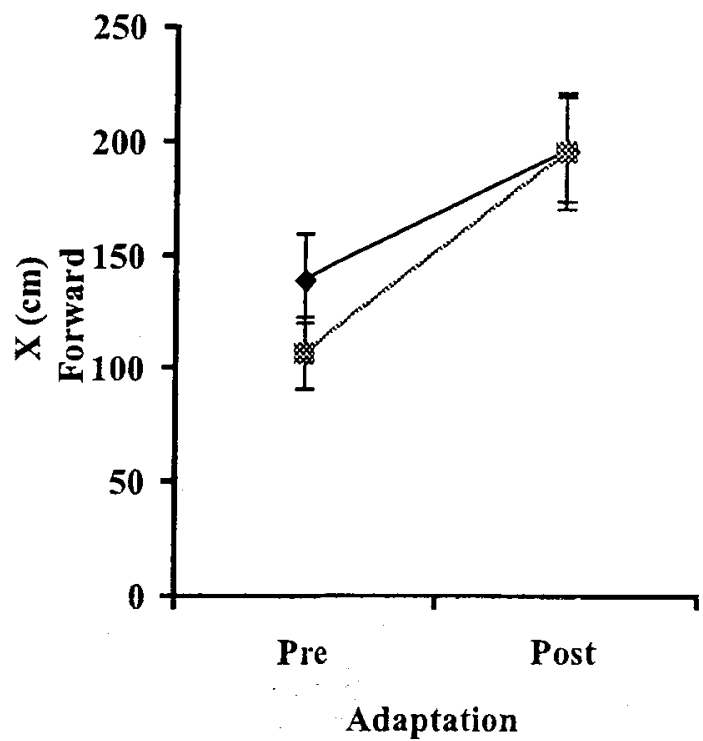

Figure 3 

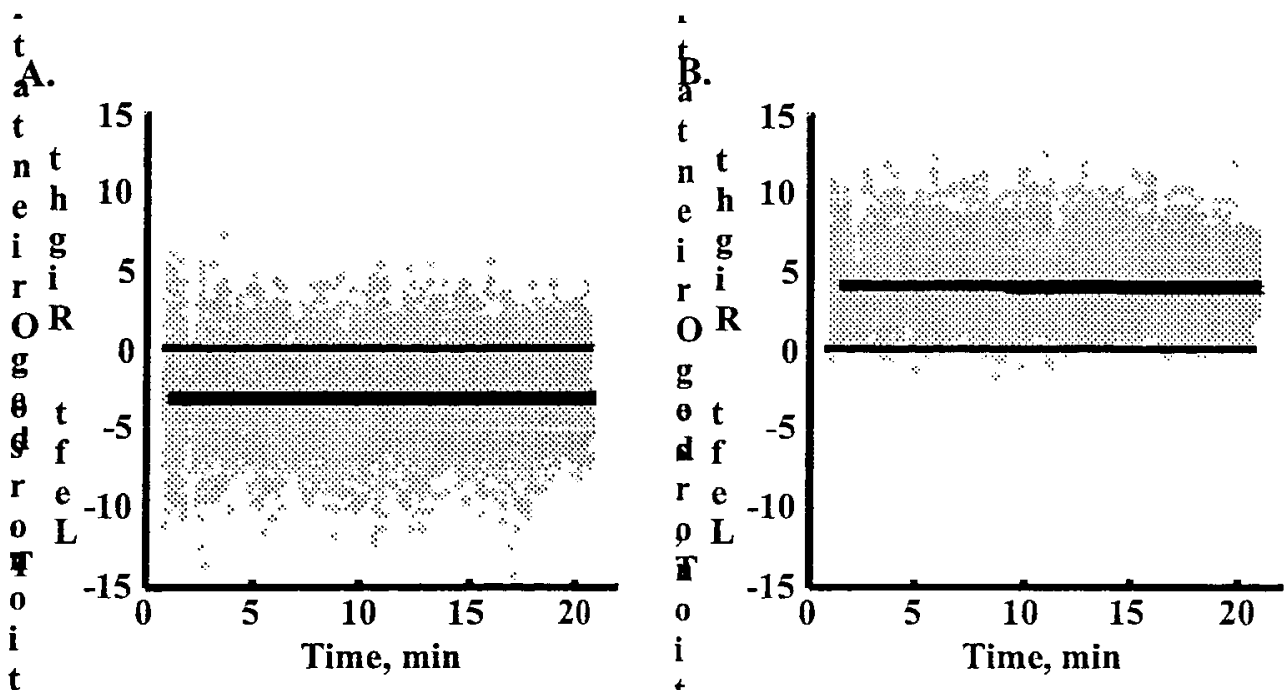

aC.

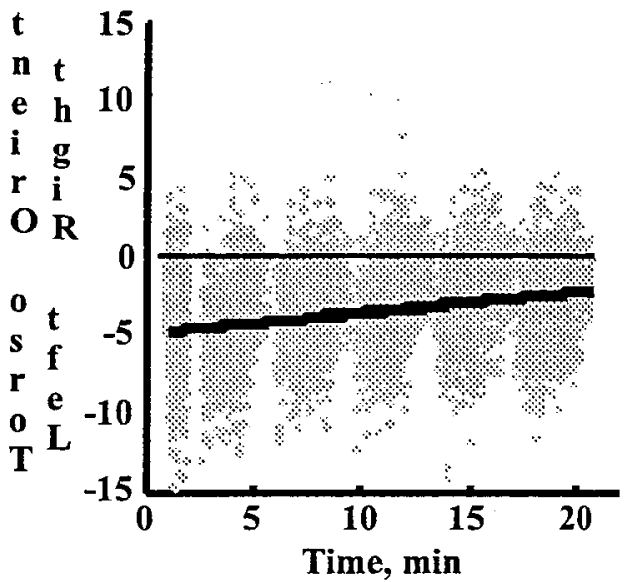

p.

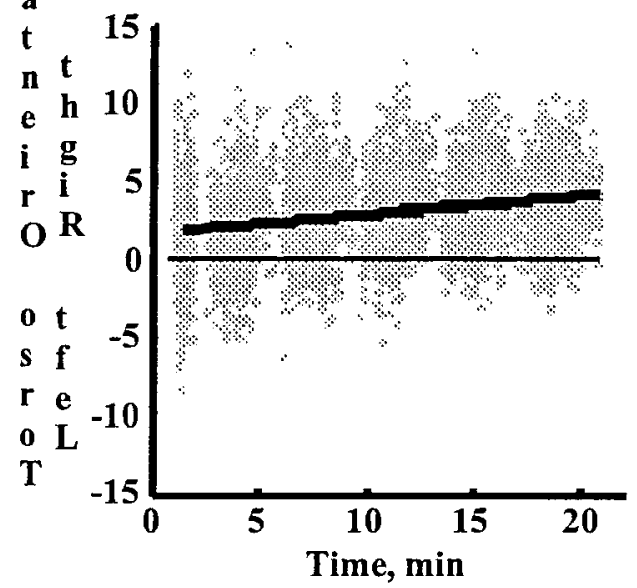

Figure 4 


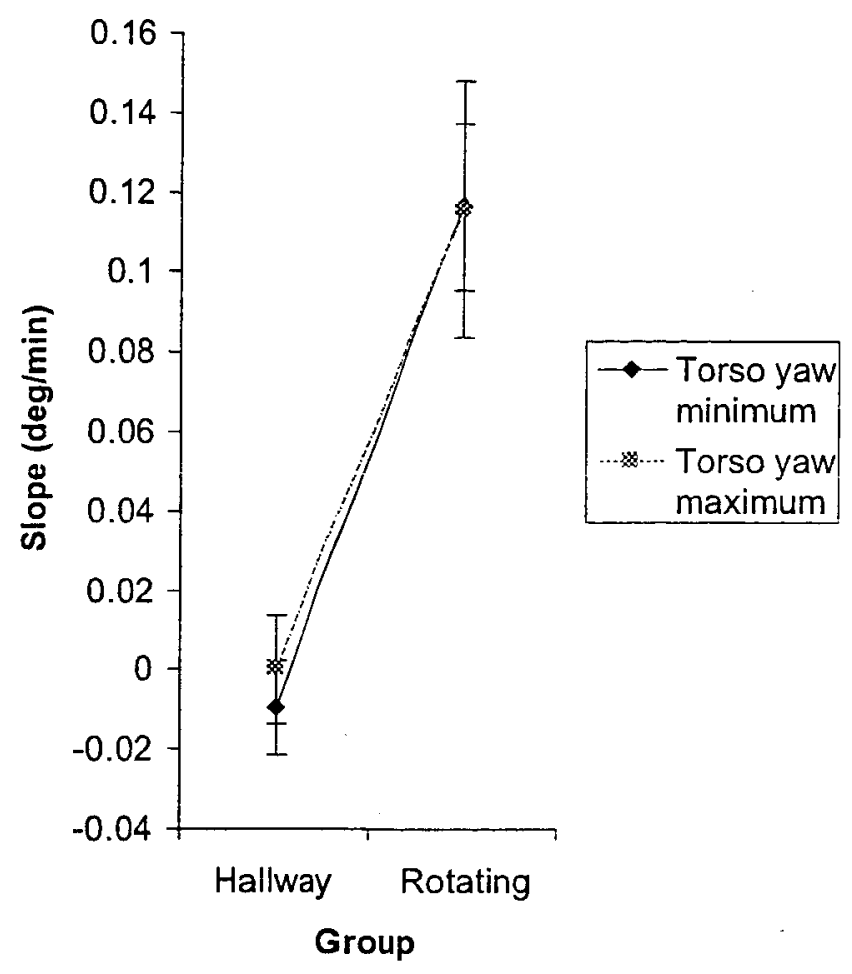

Figure 5 